\title{
Foreword by Prof. Dr. Alexander Fay
}

Almost twenty years ago, the idea of an object-oriented, manufacturer-neutral, computer-readable description of industrial plants was first presented: CAEX, short for Computer Aided Engineering eXchange ${ }^{1}$. In a world of document-oriented engineering tools, this opened up the possibility of loss-free information exchange. Soon afterwards, when internationally standardized in IEC 62424, the concept of a system model structured hierarchically with objects found more and more supporters. CAEX enables both requirements and solutions for engineering tasks to be linked to the plant objects.

CAEX is more than just an XML-based exchange format for engineering data: CAEX can support the engineering workflow consistently and connect previously separate software tools and engineering tasks during the planning, implementation and operation of industrial plants. Because of these strengths, CAEX was chosen to be the backbone of AutomationML. By integrating further description means, such as COLLADA for geometries and PLCopen XML for process descriptions and control programs, AutomationML enables the linking of all planning and operational information to the assets of a system. AutomationML, thus, provides what is necessary to build a digital twin of an industrial plant.

Thereby, AutomationML offers a multitude of application areas: in addition to modelling of industrial plants, it has been applied to model different technical systems such as communication systems, building automation systems, energy supply and distribution networks, but also various kinds of diagrams. In fact, everything which can be structured into objects and links (of whatever type) between them can be modelled with AutomationML and, thus, be transferred to other software tools for further information processing.

To this day, more than 2,000 publications from industry and academia report the capabilities and advantages of AutomationML. A lot of considerations and experiences from practical applications have contributed to the development of AutomationML, which now is available in Edition 2 and further improves the modelling capabilities and reduces the modelling effort. Therefore, it is the right time now for this book, to introduce these advantages to a wider audience. Though the basic principles of AutomationML are easy to grasp, a more detailed study of the topic is necessary to fully benefit from the advantages of AutomationML.

This book is ideal reading both for the AutomationML beginner, who will be introduced gently to the main concepts, as well as the AutomationML expert, who will find valuable modelling techniques. AutomationML can significantly simplify

1 M. Fedai, U. Epple, R. Drath, A. Fay: A Metamodel for generic data exchange between various CAE systems. "MathMod 2003" - Vienna International Conference on Mathematical Modelling, ISBN 3901608-24-9, pp. 1247-1255. 
information exchange between software tools throughout the entire life cycle of a plant, for the benefit of engineering service providers, plant constructors, plant operators and manufacturers. This book is highly recommended to all who want to know how they can participate in this development.

Prof. Dr.-Ing. Alexander Fay, Hamburg, November 2020

Helmut Schmidt University, Hamburg 